\title{
NOVEL ORGANIZATION OF MICROTUBULES IN CULTURED CENTRAL NERVOUS SYSTEM NEURONS: FORMATION OF HAIRPIN LOOPS AT ENDS OF MATURING NEURITES ${ }^{1}$
}

\author{
HO-CHING TIFFANY TSUI,* KAREN L. LANKFORD, $\ddagger$ HANS RIS,§ AND WILLIAM L. KLEIN* ${ }^{2}$ \\ * Interdepartmental Graduate Program in Neuroscience and $\ddagger$ Department of Neurobiology and Physiology, Northwestern \\ University, Evanston, Illinois 60201, and \$Department of Zoology, University of Wisconsin-Madison, Madison, Wisconsin 53706
}

Received February 16, 1984; Revised June 4, 1984; Accepted June 29, 1984

\begin{abstract}
Using the high voltage electron microscope, we have examined cultured embryonic neurons in order to understand better the organization of microtubules in developing neurites. We found that, in embryonic chick retina neurons, microtubules were abundant in the ends of neurites and showed an unusual pattern of organization. Most striking was the presence of microtubule loops; after entering the flattened region of a growth cone, microtubules frequently made tight $180^{\circ}$ turns. Occasionally these looping microtubules re-entered the neurite and returned in the direction of the cell body. Positive identification of the loop structures as microtubules was made by specific immunocytochemical labeling. Quantitative analysis showed that more than half of the retina neurons that were dissociated on embryonic day 8 and kept in culture for 4 to 6 days (E8C4 and E8C6) contained at least one microtubule that made a $180^{\circ}$ turn at flat regions along or at the tips of neurites. The area within the loops typically contained larger membranous organelles, whereas only small vesicles were seen outside the loops. Fine filaments were seen to interconnect the loops at various places, suggesting the possibility that they played a role in maintaining the shape of microtubule loops. Examination of other neurons showed that tight microtubule loops were prominent in chick spinal cord neurons, but they were rarely seen in neurons of the sympathetic ganglia or dorsal root ganglia or in NG108-15 cloned cells. Developmentally, no loops were observed in E8C1 retina neurons, but retina neurons dissociated from older embryos (12 days) did show loops after 1 day in culture; these data suggest that microtubule loops may be abundant around embryonic day 12 to 13 in the chick retina. The possible significance of this unusual microtubule organization to the control of neurite growth and bidirectional transport is discussed.
\end{abstract}

The regulation of neurite growth and intracellular transport is of particular importance in the developing nervous system. Immature neurites grow out from neuronal cell bodies and eventually establish distinctive, classifiable arborization patterns. Cellular components are transported to the expanded growing tips, called growth cones (Harrison, 1910; Nakai, 1956; Landis, 1983; Pfenninger and Johnson, 1983), and new membrane is added to neurites specifically at these regions (Bray, 1970; Feldman et al., 1981; Pfenninger and Maylie-Pfenninger, 1981). Important traffic also goes in the other direction, as developmental signals such as nerve growth factor (NGF) are taken up and transported back to the cell body (Campenot, 1977; Gundersen and Barrett, 1979). In order to understand the control of neurite growth, along with related processes such as the developmental regulation of intraneuronal transport and membrane assembly, it thus is important to establish the detailed structural organization at the ends of developing neurites.

\footnotetext{
${ }^{1}$ This work was supported by National Institutes of Health Grants RR00570 to the University of Wisconsin-Madison high voltage electron microscope facility and NS15299, DA02950, and NS18490 to W. L. K.

${ }^{2}$ To whom correspondence should be addressed, at Department of Neurobiology and Physiology, Northwestern University, Evanston, IL 60201 .
}

The present study focuses on the organization of microtubules at neurite tips of cultured CNS neurons.

Microtubules are generally recognized to play a role in determining cell shape (Yamada et al., 1970; Lasek and Shelanski, 1981) and mediating intracellular transport (Thoenen and Kreutzburg, 1981). The subcellular disposition of microtubules in neurons as well as their assembly and disassembly have recently received increasing attention (Lasek and Shelanski, 1981; Gray et al., 1982; Letourneau, 1982; Westrum et al., 1983). Microtubules appear to be assembled from subunits at initiation centers in nerve cell bodies (Spiegelman et al., 1979; Sharp et al., 1982). Most microtubules in axons appear to have their fast assembly end distal to the cell body (Burton and Paige, 1981; Heidemann et al., 1981; Filliatreau and Di Giamberardino, 1981a, b), whereas axoplasmic transport studies indicate that assembled microtubules move slowly as columns toward axon terminals (Hoffman and Lasek, 1975; Black and Lasek, 1980). The fate of microtubules at the distal ends of growing neurites and mature axons has not been established. Data obtained from thin section studies have been interpreted to show that microtubules are slightly curved structures in growth cones (Yamada et al., 1971; Johnston and Wessells, 1980; Landis, 1983), although immunofluorescence studies have indicated a more complex pattern (Shaw et al., 1981; Sponner and Holladay, 1981; Jacobs and Thomas, 1982). In mature 
terminals, microtubules are postulated to be disassembled and degraded (Lasek and Hoffman, 1976) since they are absent in conventional EM preparations (Peters et al., 1976).

To understand better the details of cytoskeletal organization at the ends of developing neurites, we have begun to examine cultured neurons with the high voltage electron microscope (HVEM). Due to their thinness, growth cones and flattened regions along neurites are particularly amenable to whole mount study (Tsui et al., 1983). Our current data show that CNS growth cones contain numerous microtubules; these microtubules assume a novel nonlinear form characterized by the presence of tight bends and loops. This phenomenon seems to occur at a particular embryonic age, and we suggest that this unusual organization may be important for the control of neurite growth. Our findings also raise the interesting question of how the typically linear structure of microtubules is controlled to give such a surprising configuration.

This work has been presented in preliminary form (Tsui and Klein, 1983).

\section{Materials and Methods}

Cell culture. Retina and spinal cord neurons were dissociated from 8-day (E8) chicken embryos with the use of trypsin as described (Tsui et al., 1983). Retina neurons with arborizing morphology were disso ciated from 12-day (E12) embryos with a modified method of James and Klein (1982). Briefly, retinas from these older embryos were incubated in papain $(0.5 \mathrm{mg} / \mathrm{ml}), 1 \mathrm{~mm}$ EGTA in $\mathrm{Ca}$, and $\mathrm{Mg}$-free Hanks' buffer for $30 \mathrm{~min}$ at $37^{\circ} \mathrm{C}$ and then gently triturated with a wide bore pipette. The plating medium contained $10 \%$ fetal calf serum and $5 \%$ chick embryo extract in Dulbecco's modified Eagle's medium (DMEM) for E8 cultures and 10\% fetal calf serum and $5 \%$ optic lobe extract (Hyndman and Adler, 1982) in DMEM for E12 retina cultures. After 3 days, the cells were fed with a serum-free supplemented medium (Bottenstein et al., 1980) to prevent clumping and proliferation of nonneuronal cells. Dorsal root ganglia (DRG) and sympathetic ganglia (SG) from the lumbosacral region of 8- to 11-day chick embryos were dissociated and cultured in NGF (7 S, Collaborative Research Inc.; 25 $\mathrm{ng} / \mathrm{ml}$ ) supplemented medium as described by Spooner and Holladay (1981). Primary cultured cells as well as neuron-like NG108-15 cloned cells were plated onto polylysine-treated and Formvar-coated gold grids for whole mount studies (Tsui et al., 1983).

Preparation for electron microscopy. After 1 to 7 days in culture, cells were fixed in $2.5 \%$ glutaraldehyde in $0.1 \mathrm{M}$ HEPES buffer at room temperature, postfixed in $0.1 \%$ osmium tetroxide in distilled water, stained in 1\% uranyl acetate, and dehydrated in ethanol, then either embedded in Epon-Araldite for sectioning or critical point dried through carbon dioxide for whole mounts. Detergent-extracted whole mounts were prepared using $0.15 \%$ Triton X-100 in a microtubulestabilizing extraction buffer (PHEM buffer: $60 \mathrm{~mm}$ PIPES, $25 \mathrm{~mm}$ HEPES, $10 \mathrm{~mm}$ EGTA, $2 \mathrm{~mm} \mathrm{MgCl}_{2}, \mathrm{pH}$ 6.9) (Schliwa and van Blerkom, 1981) at room temperature before fixation and then were stained, dehydrated, and critical point dried as untreated whole mounts. For quantitative analysis of the frequency of microtubule loop structures, pictures were taken of all of the neurite ends present on two separate preparations and were examined at a final magnification of $x$ 30,000 . Neurite ends that contained at least one microtubule making a $180^{\circ}$ turn were counted as positive. A total of 93 neurites were used in this analysis.

Immunocytochemical localization of tubulin. Retina cultures were extracted at room temperature with $0.15 \%$ Triton X-100 in PHEM buffer, fixed in $1 \%$ glutaraldehyde in PHEM buffer for $10 \mathrm{~min}$ at room temperature, treated with $0.5 \mathrm{mg} / \mathrm{ml}$ of sodium borohydride (Osborn et al., 1978) in phosphate-buffered saline (PBS) twice for $4 \mathrm{~min}$ each, and washed three times with PBS for 10 min each. The cultures were then incubated with rabbit anti-tubulin sera (Miles Laboratories, Inc.; directed against purified tubulin from 13-day chick embryo brain) at 1:10 dilution for $45 \mathrm{~min}$ at $37^{\circ} \mathrm{C}$, washed with PBS three times for $10 \mathrm{~min}$ each, and then incubated with ferritin-conjugated anti-rabbit IgG (Miles Laboratories, Inc.), postfixed in 1\% glutaraldehyde in PHEM, rinsed with PHEM, osmicated, dehydrated, and critical point dried as unlabeled preparations. Control experiments were performed using nonimmune rabbit serum in place of anti-tubulin serum.

HVEM and EM examination. Thick sections and untreated whole mounts were examined and photographed with the $1-\mathrm{MeV}$ microscope at the High Voltage Electron Microscope (HVEM) facility at the University of Wisconsin-Madison. Detergent-extracted anti-tubulin labeled and unlabeled whole mounts were examined with a JEOL $100 \mathrm{CX}$ electron microscope at $100 \mathrm{KV}$.

\section{Results}

Culture appearance of chick retina neurons. Cultures of dissociated cells from 8 -day-old chicken retina contained primarily well attached, process-bearing neurons. Tape-recorded observation using differential interference contrast microscopy with a $\times 100$ oil-immersion lens showed that neurons dissociated on $\mathrm{E} 8$ and kept in culture for 1 day invariably displayed fast neurite growth and active filopodial activity at the growth cone regions. Neurons in culture for 4 to 6 days, however, showed varied patterns of activity. Whereas some E8C4 and E8C6 neurons still showed filopodial activity and slow neurite growth, other neurons had quiescent neurite tips with very little filopodial activity and no neurite extension. However, many of the apparently inactive neurites still had enlarged structures at their tips that resembled growth cones of younger cultures. We shall refer to most enlarged regions at the end of neurites examined with the electron microscope as "neurite tips" even though many of them could have exhibited typical growth cone activity.

Occurrence of microtubule loop structures at neurite tips of cultured retina neurons. Monolayer cultures of the chick retina were processed in three different ways for electron microscopy. Thick sections and whole mounts were used for high voltage electron microscopy and Triton X-100-extracted whole mounts were used for conventional electron microscopy. Loops of microtubules were frequently observed at the ends of neurites in all three preparations.

Figure 1 shows a thick section $(0.25 \mu \mathrm{m})$ from a neurite tip of an E8C6 retina cell culture. This region contains a hairpinshaped bundle of linear elements, each 20 to $25 \mathrm{~nm}$ in width. Based on their size and appearance, these structures and the similar structures shown below in whole mounts are presumed to be microtubules. Supporting immunocytochemical evidence will be presented later. In Figure 1, a bundle of microtubules appears to enter the flat terminal region of the neurite, form a loop, and return toward the cell body. Within the neurite proper, the two parts of the bundle remain separate within the field of the section. Although the loop as a whole appears continuous, the thick section only shows part of the neurite tip, making it hard to trace the path of an individual microtubule completely. Although unlikely, Figure 1 also could be interpreted as showing two separate bundles of terminating microtubules that interdigitate.

When whole mount preparations rather than thick sections were examined, a similar pattern of microtubules could be seen. Whole mount preparations showed complete three-dimensional organization of all organelles in thin areas of neurites and growth cones and hence were particularly suitable for the study of the overall organization of microtubules. In Figure $2 A$, microtubule bundles in the neurite enter the flattened region at the end of the neurite and loop around in this region. Microtubules may make wide U-shaped turns (Fig. $2 B$ ) or small circular turns (Fig. $2 C$ ) at these regions. When viewed in stereo (Fig. 2B), the looping microtubules seem to trap the larger membranous organelles inside the loops. A more detailed examination of the relationship between the microtubules and membranous organelles is presented later. The looping pattern was also present at occasional flat regions along neurites. Some of these flat regions appeared as branching points of neurites, as in Figure $2 D$. In these areas, some microtubules remained straight and continued in two different directions, whereas others made hook-shaped structures. Overall, a general looping 

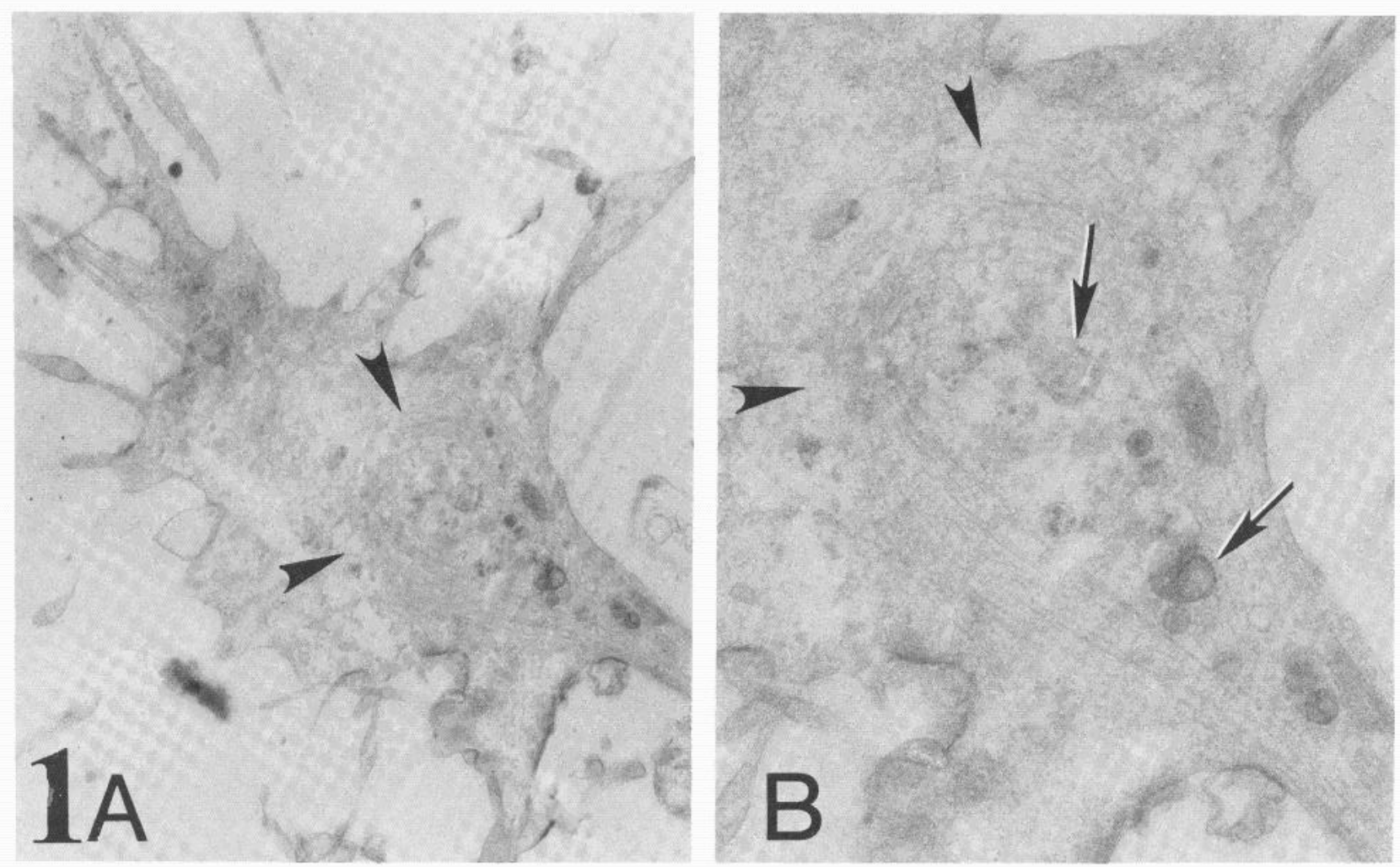

Figure 1. Thick section from a neurite tip of an E8C6 retina neuron. Note the prominent bundle of linear elements, 20 to $25 \mathrm{~nm}$ in width, forming a hairpin-shaped loop (arrowheads). Large membranous organelles (arrows) are present inside the loop area but not outside the loop. Magnification: $A, \times 7,000 ; B, \times 14,000$.

pattern of microtubules was readily apparent in whole mount preparations.

Since it was not possible to trace complete paths of individual microtubules in sectioned materials and in untreated whole mounts, some cultures were treated with Triton X-100 in a microtubule-stabilizing buffer according to the method of Schliwa and van Blerkom (1981) (Figs. 3 and 4). This procedure is known to remove the membrane and less stable cytoplasmic components, leaving a clear image of the cytoskeleton. Extracted neurons typically contained the nucleus and various cytoskeletal elements in the neurites.

EM observation of extracted E8C4 and E8C6 neurons showed that the organization of microtubules in neurite tips exhibited a spectrum of complexity. In some neurite tips, microtubules spread out but remained as straight filaments (Fig. $3 A$ ). However, in other neurite tips, while some microtubules remained straight, other microtubules were seen to make $180^{\circ}$ turns within the flat region (Fig. $3 D$; see also Fig. $4 B$ ). Very often (Fig. 3, $B$ and $C$ ), swirls of microtubules could be seen in these neurite terminal regions, giving the impression that each microtubule turned around many times at the terminal region of the neurite.

Some microtubules made hairpin loops at the terminal flat regions and re-entered the neurite. This phenomenon is most clearly demonstrated in Figure 4. In this figure, a bundle of microtubules can be seen to spread out and bend around at region $a$. Individual microtubules then either turn back toward the cell body or continue in the original direction. Some of the microtubules terminate further away from the cell body at region $b$. However, at this region (Fig. $4, B$ and $C$ ), two microtubules can be seen clearly to turn back and join the main bundle of microtubules in the neurite.

Because of the relative ease in tracing these individual microtubules, a quantitative analysis of the loop frequency was performed. Of the 93 neurite ends that were examined from E8C4 and E8C6 retina cultures, 49 contained at least one microtubule that made a $180^{\circ}$ turn. This analysis showed that more than half of the neurites of these cultures contained tight bends of microtubules.

Microtubules with two free ends were usually not seen in any flattened regions. This was in contrast to the short and freeended bundles of finer filaments seen outside the microtubule loop areas (Fig. $3 C$ ). Although these bundles resembled microtubules in diameter, careful examination revealed splitting of the bundle into finer filaments at the ends. Furthermore, these bundles were not labeled with anti-tubulin antibodies as described below.

To confirm that the 20-nm looping structures were indeed microtubules, we labeled Triton X-100-extracted cultures with rabbit antiserum directed against chick brain tubulin and ferritin-conjugated anti-rabbit IgG. In these preparations, the looping filaments were decorated with the antibodies and appeared as $45-\mathrm{nm}$ structures (Fig. 3, $C$ and $D$ ). This diameter is reasonable for a microtubule of diameter $20 \mathrm{~nm}$ decorated around its circumference by two layers of antibody molecules, since a single IgG has a diameter of about $9 \mathrm{~nm}$ (Osborn et al., 1978). Grains of ferritin in the antibodies could be seen at higher magnification (Fig. 5). Finer filaments that interconnected the microtubules and the bundles of filaments outside of the loop area were not labeled. Control preparations treated with nonimmune sera in place of anti-tubulin sera showed undecorated 20-nm filaments (Fig. $5 C$ ). All of the evidence taken together showed that the looping structures within neurites were microtubules.

Examination of other neurons. Extracted and unextracted neurons of chick spinal cord, DRG, and SG as well as neuronlike NG108-15 hybrid cells were examined to see whether looping microtubules were present in neurons other than retina 


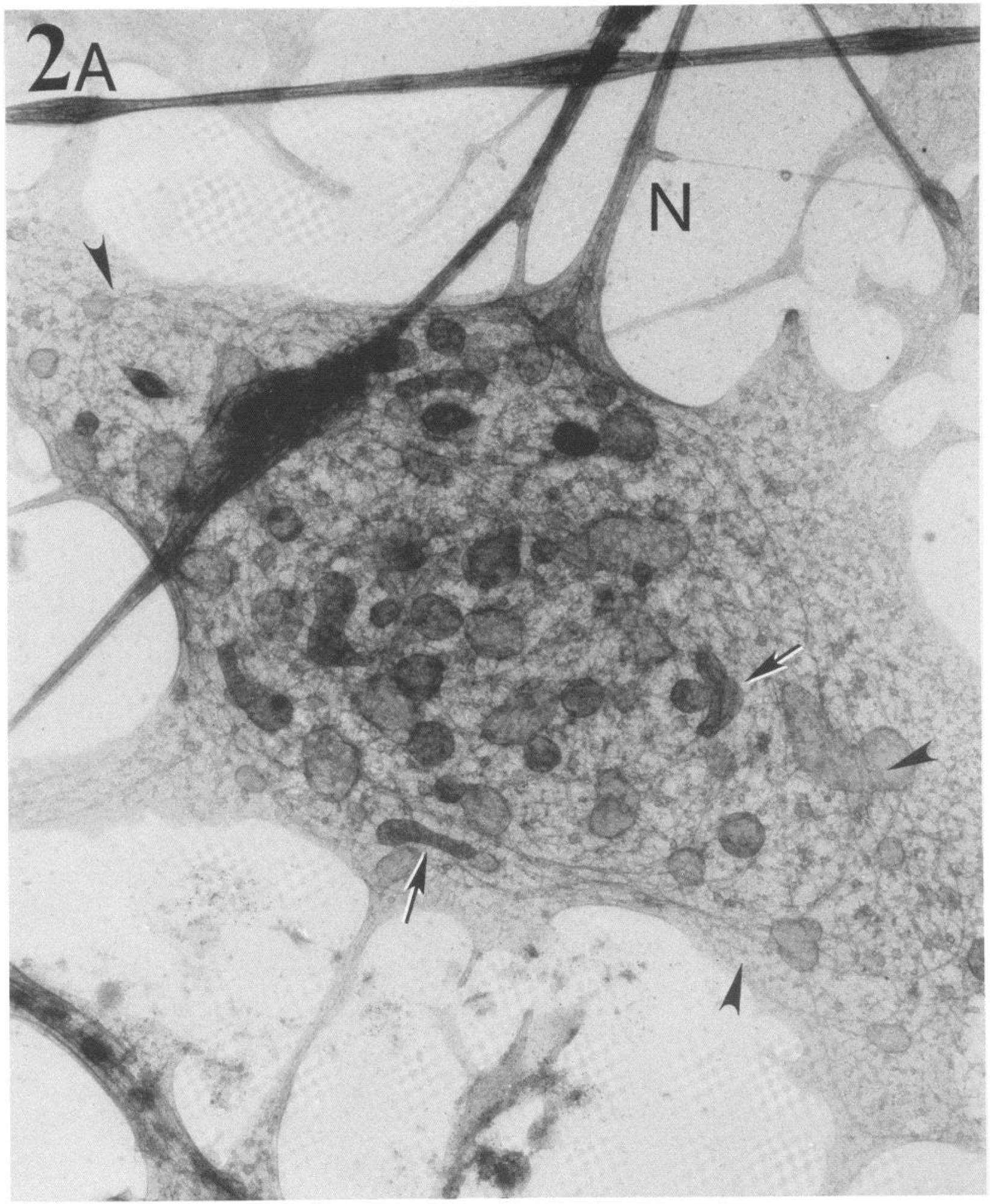

Figure 2. Unextracted whole mount retina neurons showing loops of microtubules and their relationship to other organelles. $A$, E8C6 retina neuron. Microtubules from the neurite $(N)$ turn around (arrowheads) in the flat area at the end of the neurite. Elongated organelles, possibly mitochondria (arrows), are seen to follow the general curvature of the microtubules. Note the many bigger membranous sacs and vesicles inside the microtubule coils and only smaller vesicles enmeshed in a filamentous network outside the loop area. Magnification $\times 20,000$. B, Stereo pair of an E8C3 retina neuron. Microtubules make U-shaped turns at the end of the neurite. When viewed in stereo, the large membranous organelles appear to be trapped inside the microtubule loops. Magnification $\times 20,000$. C, Stereo pair of an E8C3 retina neuron showing distinct circular configuration of microtubules (arrowheads). Magnification $\times 20,000$. D. A flat branching region of an E8C3 retina neuron. Most of the microtubules from the neurite (lower left corner) continue as relatively straight structures in two directions. However, curved microtubules (arrowheads) can be seen at the center of the flat region, and they seem to enclose large membranous organelles. There is extensive cross-linkage among various membranous organelles and the microtubules through a network of fine ( 5 to $9 \mathrm{~nm}$ ), discrete, nontapering filaments. At an area lacking microtubules (asterisk), other membranous organelles also are absent. Magnification $\times 34,500$. 

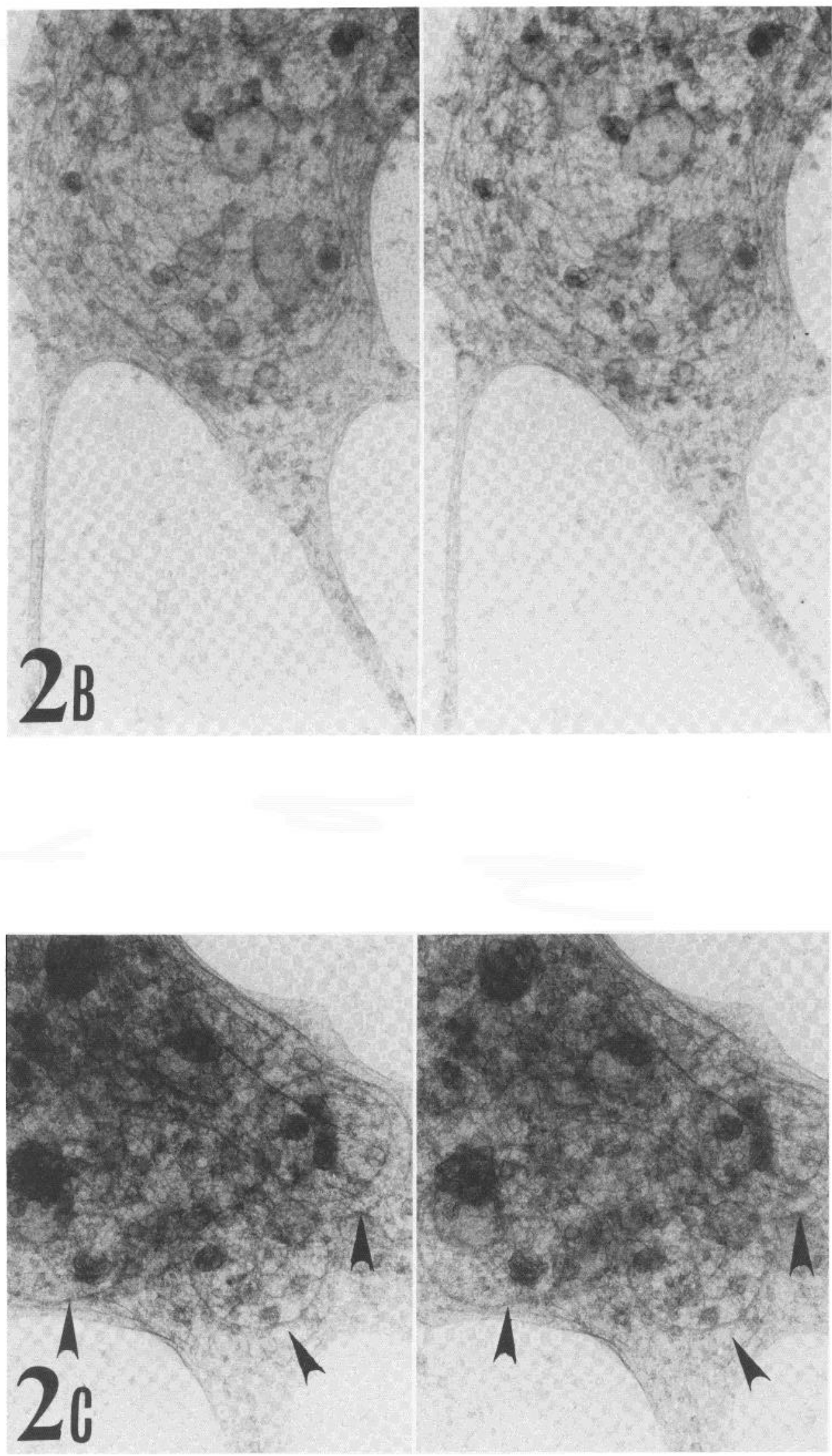

Figure 2, B and C 


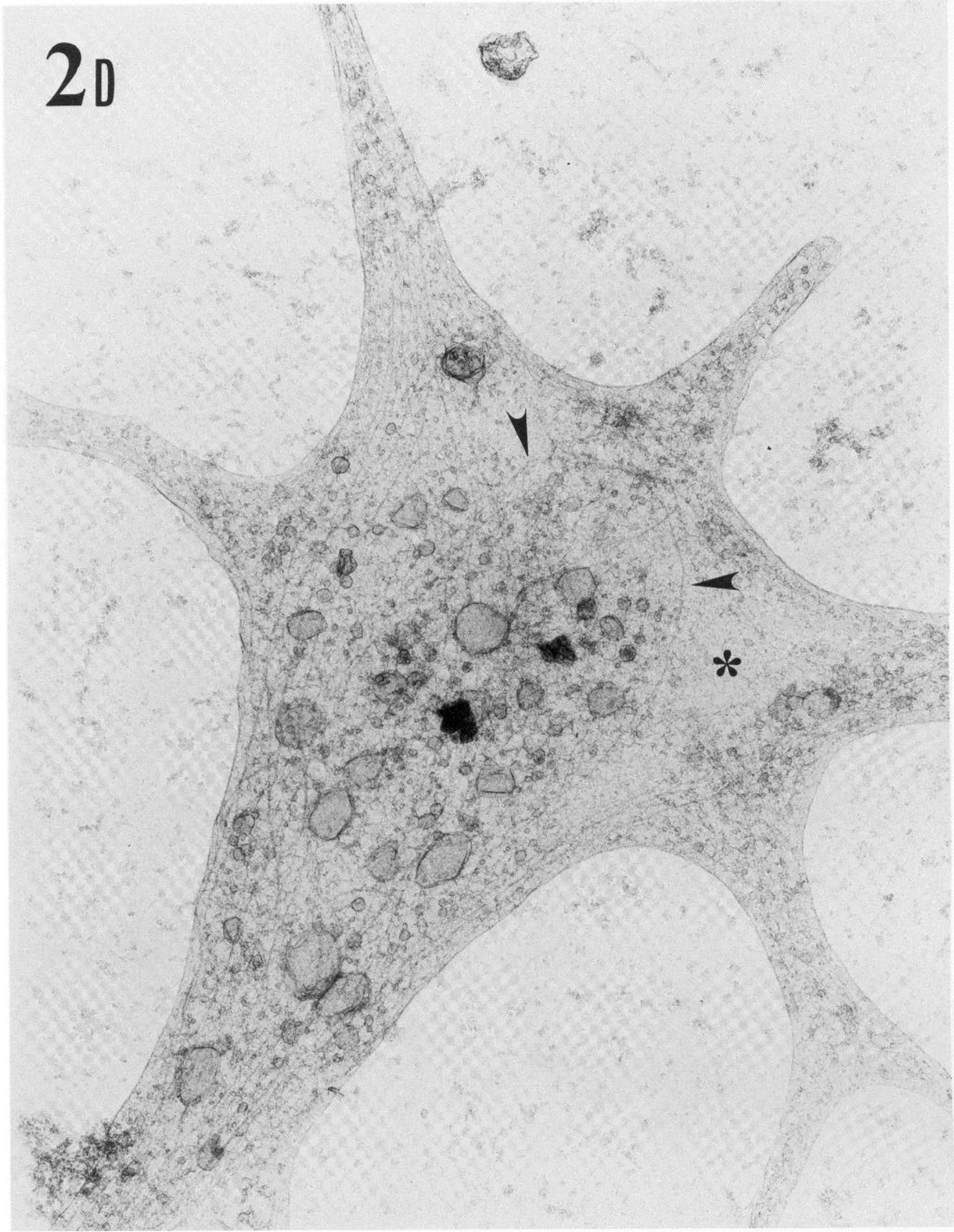

Figure $2 D$ 

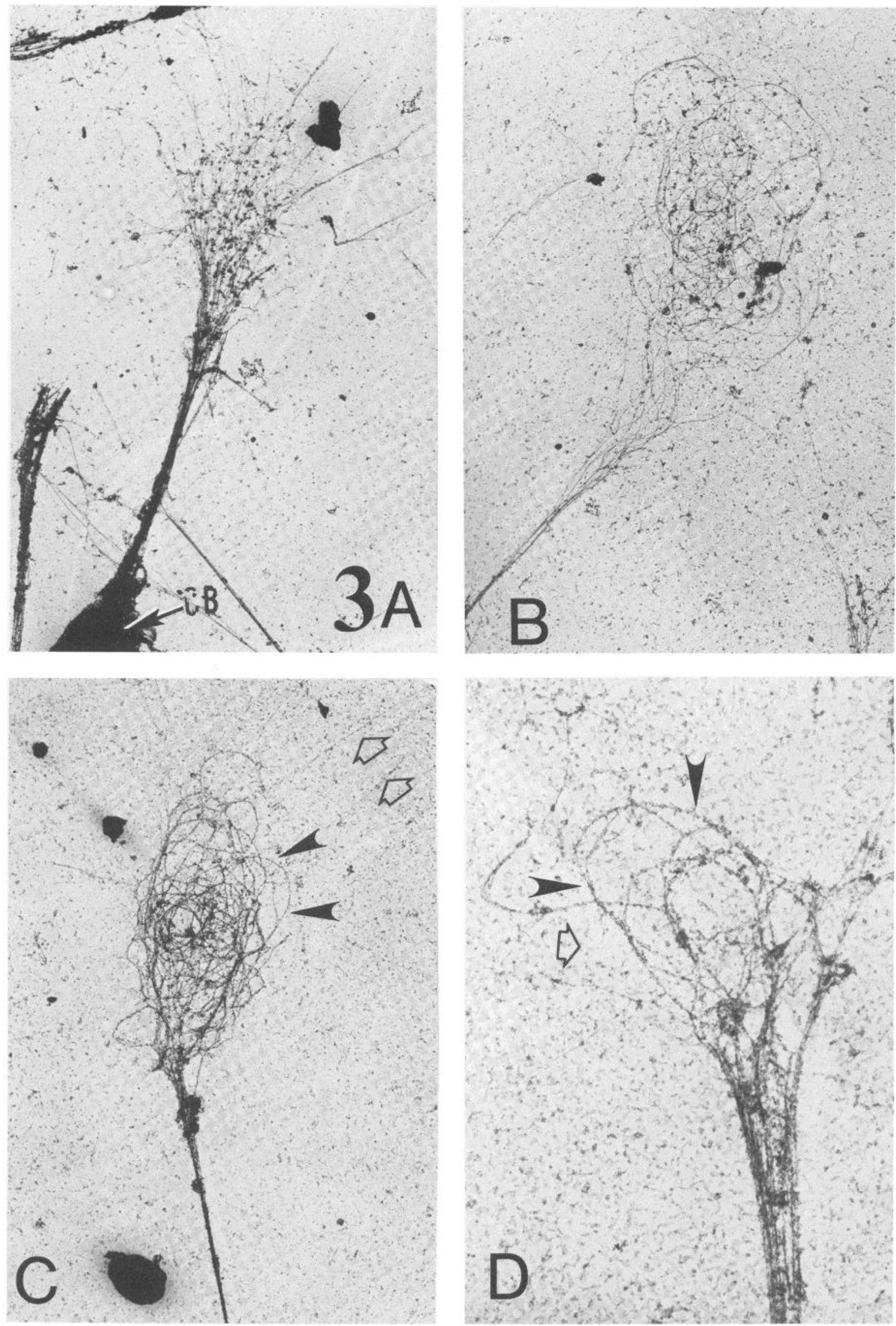
cells. I ooping patterns were found in $\mathrm{F} 8 \mathrm{C}, 2$ spinal cord neurons (not shown) whereas microtubules in DRG, SG, and NG108 cells were only slightly curvy at the ends of neurites, rarely showing any $180^{\circ}$ turns.

Association of microtubules with other filaments. A network of fine filaments was observed throughout the neurites and growth cones in unextracted whole mount preparations (Fig. 2). In Triton X-100-extracted preparations, much of the network was absent, but distinct thin (6 to $10 \mathrm{~nm}$ in diameter) and shorter (60 to $400 \mathrm{~nm}$ ) filaments were seen to interconnect microtubules at various places (Figs. 4 and 5 ). These filaments seemed to be under tension and, therefore, gave the appearance of holding the microtubules in their shape.

Association of microtubules with membranous organelles. Extensive cross-linkages were found between microtubules and various membranous organelles in our unextracted whole mount preparations. In the center of the growth cone there were large membranous sacs (up to $400 \mathrm{~nm}$ ) and vesicles of irregular sizes ( 25 to $75 \mathrm{~nm}$ ) (Fig. 2). These membranous organelles were connected to microtubules through a network of fine $(5$ to $9 \mathrm{~nm}$ ), discrete, nontapering filaments (Fig. $2 D$ ). Microtubule-membranous organelle interaction is also indirectly illustrated by the curving pattern of the elongated organelles (Fig. $2 A$ ). These elongated organelles, presumably mitochondria, often followed the general curvature of the looping microtubules.

Large membranous sacs and bigger vesicles also seemed to be encaged in the microtubule loops (Fig. 2). In contrast, outside the loop area, there were usually only small vesicles interconnected in a filamentous network.

Developmental studies of microtubule organization in neurites and growth cones of retina neurons. In cultures of cells obtained from $\mathrm{E} 8$ retinas, loops of microtubules were more prevalent in older cultures ( 4 to 6 days) than in younger cultures (1 to 2 days). No tight loops have ever been seen in $\mathrm{E} 8 \mathrm{C} 1$ cultures (Fig. 6A). To determine whether microtubule loops were induced by culture conditions or were developed at a particular stage of embryogenesis, we also examined arborized and viable retina neurons obtained from 12-day-old chick embryos after they were cultured for 1 day. In contrast to $\mathrm{E} 8 \mathrm{C} 1$ retina neurons, many E12C1 neurons contained looping microtubules (Fig. 6, $B$ and $C$ ). Some of the loops in E12C1 neurons appeared, not in conventional growth cone structures, but, rather, at slightly widened regions of neurites (Fig. $6 \mathrm{C}$ ). Since the microtubule loops were abundant around $\mathrm{E} 13$ regardless of whether the cells were put in culture on day 8 or day 12 , it is more likely that microtubule loops were formed at a particular embryonic age rather than after a particular time in culture.

\section{Discussion}

The evidence presented here shows that microtubules do not always terminate abruptly at the ends of CNS neurites but often make tight $180^{\circ}$ turns. Occasionally these microtubules re-enter the neurite after making a hairpin-shaped loop. This finding is somewhat unexpected, considering the generally accepted views that microtubules appear as straight or slightly curved structures at growth cones (Yamada et al., 1971; Johnston and Wessells, 1980; Landis, 1983) and that microtubules are disassembled at nerve terminals of mature animals (Hoff- man and Iasek, 1975; I asek and Hoffman, 1976; Black and Lasek, 1980). Since the same looping structures were seen in thick sections, extracted cells, and complete whole mounts, it is very unlikely that they were artifacts of preparation. These looping microtubules were not of rare occurrence as more than half of the neurite tips of $\mathrm{E} 8 \mathrm{C} 4$ retina neurons contained at least one microtubule that originated from the neurite and made $180^{\circ}$ turns at the neurite terminal. In retina neurons, these loops became abundant by E13, irrespective of whether the cells were put in culture on day 8 or day 12 , indicating a possible developmental function.

Comparison with previous studies. Our ability to observe microtubule loops at the ends of neurites probably is due to the use of EM whole mount techniques as well as the use of CNS neurons for study. Because of their large size, loops of microtubules would appear as fragments in conventional thin section studies (Yamada et al., 1971; Johnston and Wessells, 1980). Previous immunofluorescence studies (Shaw et al., 1981; Spooner and Holladay, 1981; Jacobs and Thomas, 1982) did show a more complete and complicated pattern of microtubules in growth cones of DRG neurons. However, the resolution of such studies is too low to show the configuration of individual microtubules. The occurrence of such loops appears to vary among neuron types. Although loops were abundant in retina and spinal cord cultures, cultures of peripheral neurons, which were used for most previous studies (Bray and Bunge, 1981; Letourneau, 1982, 1983; Tosney and Wessells, 1983), rarely contained tight loops of microtubules.

Mechanism of loop formation. An important and as yet unsolved question raised by the occurrence of loops is what causes the change in direction that microtubules take. Microtubules in cultured neurons can make a $180^{\circ}$ turn within a diameter of $0.5 \mu \mathrm{m}$. Microtubule assembly from tubulin in vitro (Summers and Kirschner, 1979; Telzer and Rosenbaum, 1980), however, gives only long straight structures, although modest bends in microtubules in living cells (Schliwa and van Blerkom, 1981; Nemhauser et al., 1983) are commonly observed. Microtubuleassociated regulatory proteins may play a role. Present data are consistent with the idea that bends of microtubules may be imposed by interaction with other cytoskeletal structures. We have observed the presence of many finer and shorter filaments at various parts of the loops, seemingly tying the loops together and preserving their shape. Another possibility is that the loops are formed as a result of favored microtubule polymerization conditions at the neurite tip region. This is consistent with a recent study (Letourneau and Ressler, 1984) in which similar microtubule loops were induced in the cultured DRG neurons with the use of taxol, which promotes polymerization of tubulin.

Functional significance of the loops. Microtubules have been postulated to play an important role in intracellular transport (Ochs, 1972; Schwartz et al., 1976; Thoenen and Kreutzburg, 1981). Conceivably, loops of microtubules may be involved in regulating the direction or amount of transport. This function could be particularly important in growth cones where there is a high rate of membrane turnover (Feldman et al., 1981; Pfenninger and Maylie-Pfenninger, 1981). A role for the loops in controlling the disposition of intracellular membranous structures is consistent with three types of observations made with the HVEM: (1) elongated organelles follow the general curva-

Figure 3. Cytoskeleton of cultured E8C6 neurons after Triton X-100 extraction showing various forms of microtubule organization at the ends of neurites. $A$, Straight microtubules at the end of a neurite whose cell body $(C B)$ is situated at the lower left corner. Magnification $\times 7,000$. $B$, Swirls of microtubules at the end of a neurite. Microtubules appear to turn around many times at this region. Magnification $\times 8,000$. $C$ and $D$, Anti-tubulin-labeled neurites of retina neurons showing loops of microtubules at neurite ends. Extracted cultures were incubated with rabbit antiserum directed against chick brain tubulin and ferritin-conjugated anti-rabbit IgG as described under "Materials and Methods." The looping structures appear as thicker $(45 \mathrm{~nm})$ filaments. This diameter is reasonable for a microtubule of diameter $20 \mathrm{~nm}$ decorated around its circumference by two layers of antibody molecules. In contrast, discontinuous filaments outside of the loop areas (open arrows) and finer filaments within the loop areas are not labeled. Parts of the loop areas in $C$ and $D$ (arrowheads) are shown in higher magnification in Figure 5 , $A$ and $B$, respectively. Magnification: $C, \times 7,000 ; D, \times 15,000$. 

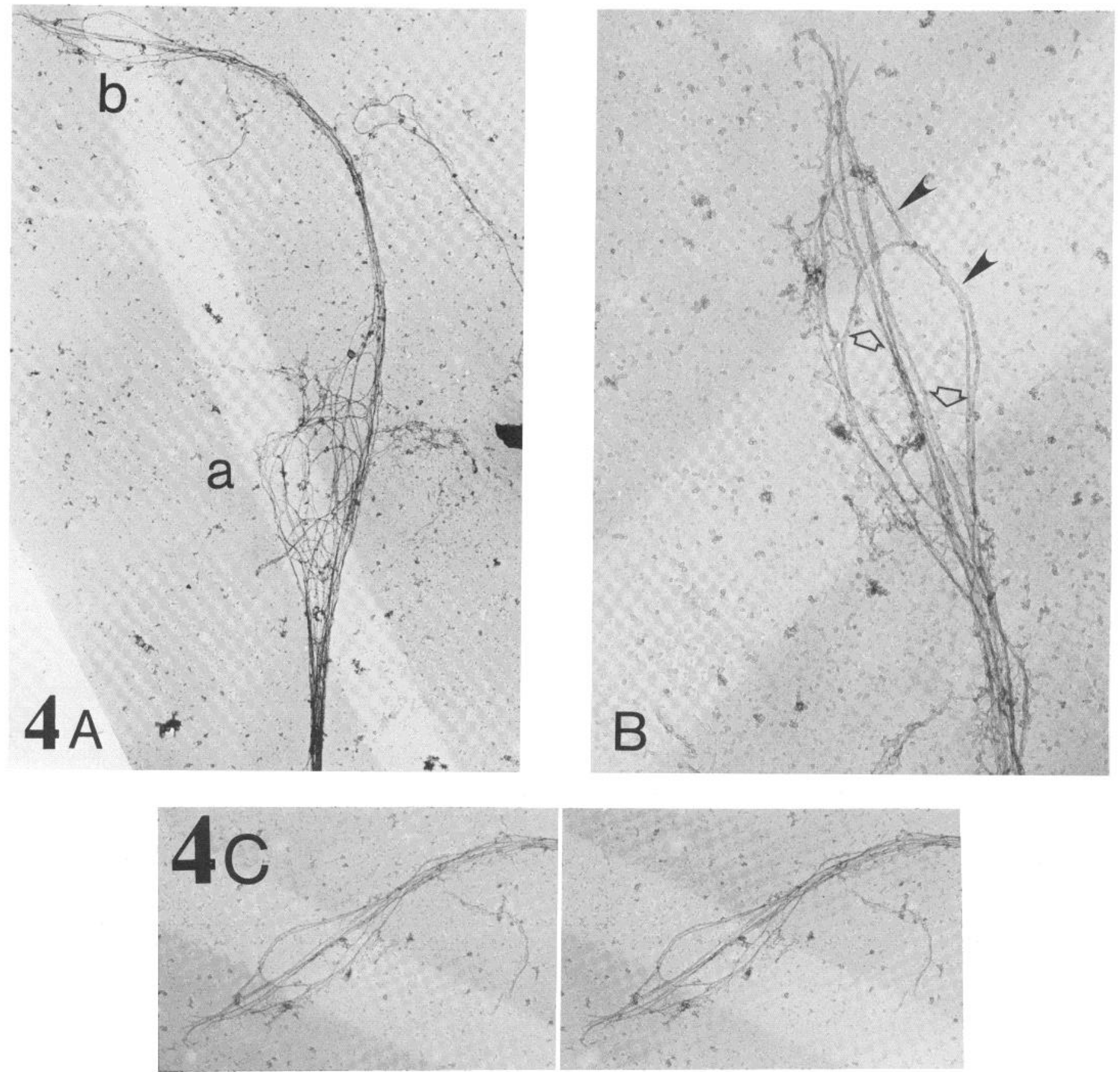

Figure 4. Hairpin-shaped microtubules at a flat region and at the end of a neurite. $A, \mathrm{~A}$ bundle of microtubules in the neurite can be seen to spread out and bend around at flat region $a$. Individual elements either turn back toward the cell body or continue in the original direction. Further away from the cell body at region $b$, two microtubules turn back to form hairpin loops while others end at this region. Area $b$ is shown at higher magnification in $B$. Magnification $\times 10,400$. B. High magnification of area $b$, seen in $A$, showing hairpin loops of microtubules and their interaction with finer filaments. Two microtubules (arrowheads) emerge from the neurite, loop around, and return to the bundle. Shorter $(60$ to $500 \mathrm{~nm})$ and finer $(6$ to $10 \mathrm{~nm})$ filaments (open arrows) are seen to connect the microtubules. Magnification $\times 32,000$. C, A stereo view of $B$, shown to facilitate the tracing of individual microtubules. Magnification $\times 14,000$.

ture of looping microtubules in growth cones (Fig. $2 A$ ), (2) extensive interconnection is present between microtubules and membranous organelles (Tsui et al., 1983), and (3) larger membranous organelles appear to be encaged within loop areas (Fig. 2).

The restriction of larger organelles, which appear to be smooth endoplasmic reticulum and mitochondria, to areas within microtubule loop areas suggests that loop formation might play a role in restricting neurite growth. This idea is supported by the developmental data. Microtubule loops were absent in E8C1 cultures where almost all neurons showed active growth cone activity and neurite extension. However, these loops were abundant in $\mathrm{E} 8 \mathrm{C} 4$ cultures, where most neurite tips appeared relatively quiescent. Studies on both E8 and E12 cultures showed that microtubule loops were abundant in retina cultures at a time equivalent to E13. At this stage in the intact retina, neurite growth of certain neurons is in its final stages. By E13, many bipolar cells, amacrine cells, and ganglion cells have acquired their distinct morphological cell shapes (Ramón y Cajal, 1972). 

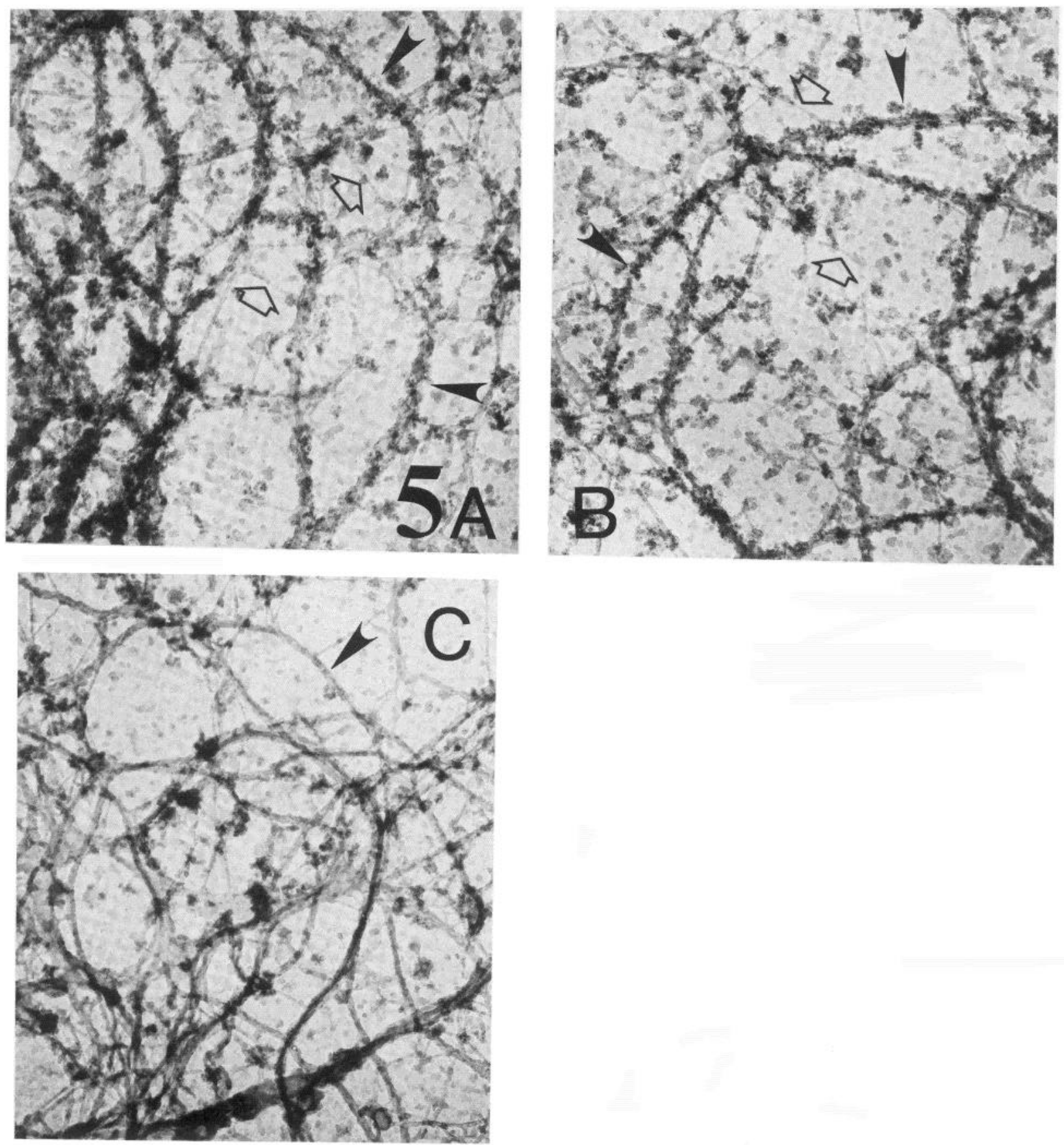

Figure 5. High magnification of looping microtubules in anti-tubulin-labeled neurons. $A$ and $B$, Parts of Figure $3, C$ and $D$, respectively, to show the labeled microtubules and the unlabeled finer filaments. Microtubules (arrowheads) are decorated with ferritin-conjugated IgGs (see "Materials and Methods"). Finer filaments (open arrows) that interconnect microtubules are not labeled. Magnification $\times 50,000$. $C$, Control preparation treated with nonimmune serum in place of anti-tubulin serum. Undecorated 20-nm looping structures (arrowhead) are present in these preparations. Magnification $\times 50,000$.

Besides blocking the flow of larger organelles, loop formation also could limit neurite growth in a second way. Microtubules are essential structures for the growth and stability of neurites. At present, there is no strong evidence indicating whether tubulin subunits are added to the microtubules at the cell body (Black and Lasek, 1980) or at the growth cone region (Burton and Paige, 1981; Heidemann et al., 1981) during development. However, if neurite growth were primarily due to the addition of tubulin subunits at the distal region, microtubule polymerization per se no longer would be expected to promote neurite elongation when loops are present. Addition of subunits at microtubule ends would not result in microtubule growth away from the cell body.

Microtubule organization at later stages of development. We have found that microtubules were not present in $\mathrm{E} 8 \mathrm{C} 1$ retina cultures but were abundant in $\mathrm{E} 8 \mathrm{C} 4$ cultures. We have not yet determined whether loops remain in neurites in later stages of development of whether they are transient structures. In older cultures (E8C6 or E12C1), some loops were found at slightly widened regions of neurites (Fig. $6 \mathrm{C}$ ). Thus, it is possible that loops that originally were in the growth cones could remain in the terminal regions of mature axons and dendrites provided they were in a thicker region, since the smallest diameter of 

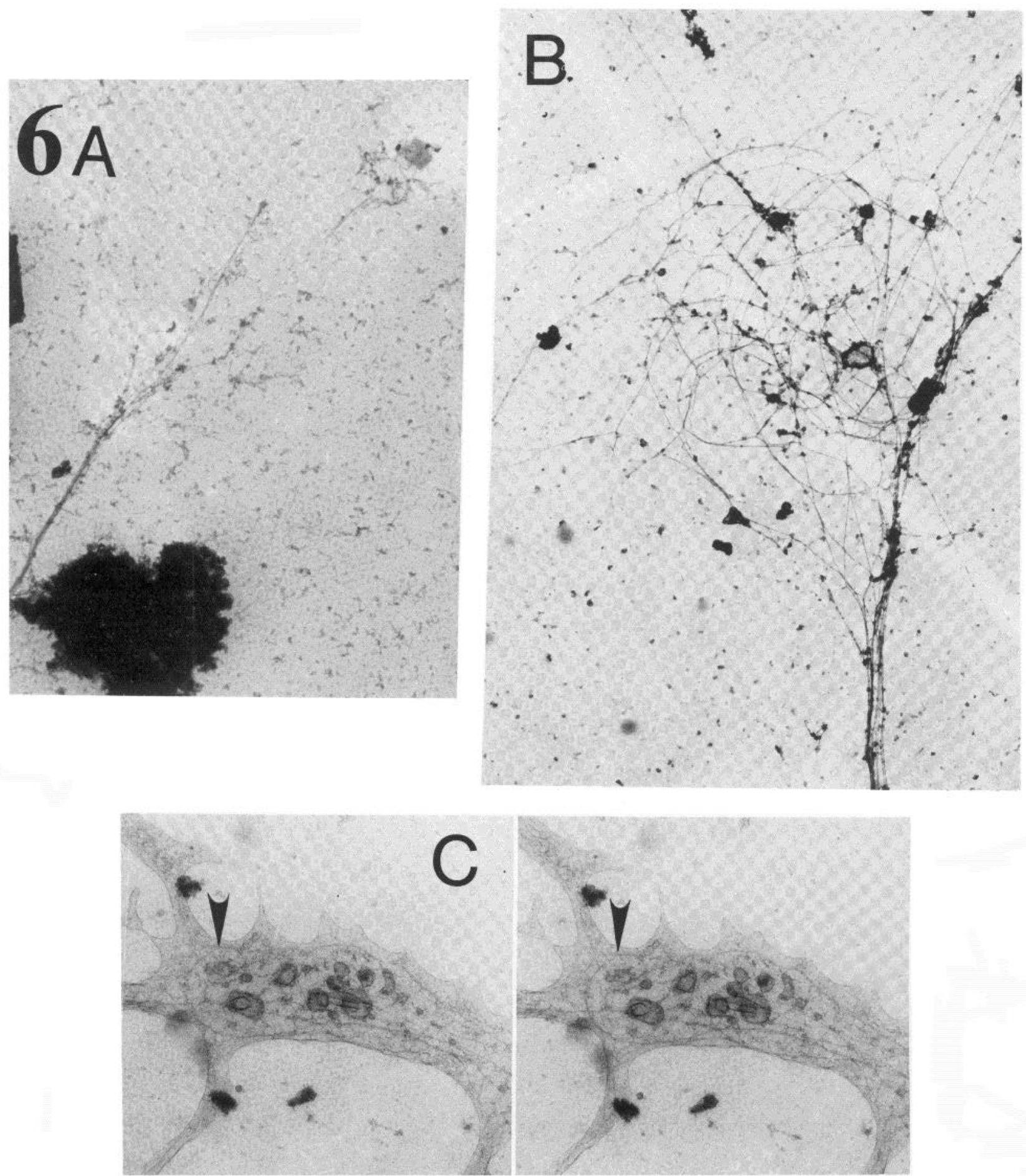

Figure 6. Differences in microtubule organization between $\mathrm{E} 8 \mathrm{C} 1$ and $\mathrm{E} 12 \mathrm{C} 1$ retina neurons. $A$, Straight microtubules in a Triton $\mathrm{X}-100$ extracted E8C1 retina growth cone. Magnification $\times 16,000$. B, Looping pattern of microtubules in a Triton X-100-extracted E12C1 retina neuron. Magnification $\times 16,000$. C, Stereo pair of an unextracted whole mount E12C1 neuron. Hairpin-shaped microtubules (arrowheads) are present in a slightly widened region of a neurite. Magnification $\times 20,000$.

the loops was $0.5 \mu \mathrm{m}$ (Fig. $4 B$ ). Alternatively, fewer loops may be present in mature animals, as suggested by the observation that microtubules in axons of mature animals are predominantly of one polarity (Burton and Paige, 1981; Filliatreau and Di Giamberardino, 1981a, b; Heidemann et al., 1981). However, the polarity data also could be explained if microtubules ended near terminals shortly after they formed loops.

To test developmental and other possible roles for microtu- bule loops, functional studies must be done along with high resolution ultrastructural analysis. We plan first to observe living neurons with the powerful VEC-DIC microscope (Allen et al., 1982; Brady et al., 1982), and then to examine the same area as whole mount under the HVEM. We are hopeful that such studies of developing CNS neurites will provide new insight into the relationship between cell structure, growth cone function, and the control of neurite growth. 


\section{References}

Allen, R. D., J. Metuzals, I. Tasaki, S. T. Brady, and S. P. Gilbert (1982) Fast axonal transport in squid giant axon. Science 218: 11271129.

Black, M. M., and R. J. Lasek (1980) Slow components of axonal transport: Two cytoskeletal networks. .J Cell Biol. 86: 616-623.

Bottenstein, J. E., S. D. Skaper, S. S. Varon, and G. H. Sato (1980) Selective survival of neurons from chick embryo sensory ganglionic dissociates utilizing serum-free supplemented medium. Exp. Cell. Res. 125: 183-190.

Brady, S. T., R. J. Lasek, and R. D. Allen (1982) Fast axonal transport in extruded axoplasm from squid giant axon. Science 218: 1129-1131.

Bray, D. (1970) Surface movements during the growth of single explanted neurons. Proc. Natl. Acad. Sci. U. S. A. 65: 905-910.

Bray, D., and M. B. Bunge (1981) Serial analysis of microtubules in cultured rat sensory axons. J. Neurocytol. 10: 589-605.

Burton, P. R., and J. L. Paige (1981) Polarity of axoplasmic microtubules in the olfactory nerve of the frog. Proc. Natl. Acad. Sci. U. S. A. 78: 3269-3273.

Campenot, R. B. (1977) Local control of neurite development by nerve growth factor. Proc. Natl. Acad. Sci. U. S. A. 74: 4516-4519.

Feldman, E. L., D. Axelrod, M. Schwartz, A. M. Heacock, and B. W. Agranoff (1981) Studies on the localization of newly added membrane in growing neurites. J. Neurobiol. 12: 591-598.

Filliatreau, G., and L. Di Giamberardino (1981a) In vivo reassembly of axonal microtubules: A fast unidirectional process. Biol. Cell 41: 63 66.

Filliatreau, G., and L. Di Giamberardino (1981b) Microtubule polarity in myelinated axons as studied after decoration with tubulin. Biol. Cell 42: 69-72.

Gray, E. G., L. E. Westrum, R. D. Burgoyne, and J. Barron (1982) Synaptic organization and neuron microtubule organization. Cell Tissue Res. 226: 579-588.

Gundersen, R. W., and J. N. Barrett (1979) Neuronal chemotaxis: Chick dorsal-root axons turn toward high concentration of nerve growth factor. Science 206: 1079-1080.

Harrison, R. G. (1910) The outgrowth of nerve fibre as a mode of protoplasmic movement. J. Exp. Zool. 9: 787-846.

Heidemann, S. R., J. M. Landers, and M. A. Hamborg (1981) Polarity orientation of axonal microtubules. J. Cell Biol. 91: 661-665.

Hoffman, P. N., and R. J. Lasek (1975) The slow component of axonal transport. Identification of major structural polypeptides of the axon and their generality among mammalian neurons. J. Cell Biol. 66: $351-366$.

Hyndman, A. G., and R. Adler (1982) Neural retina development in vitro: Effects of tissue extracts on cell survival and neuritic development in purified neuronal cultures. Dev. Neurosci. 5: 40-53.

Jacobs, M., and C. Thomas (1982) The organization of $10 \mathrm{~nm}$ filaments and microtubules in embryonic neurons from spinal ganglia. J. Neurocytol. 11: 657-669.

James, W. M., and W. L. Klein (1982) Autoradiography of dendritic acetylcholine receptors. A method for study of isolated neurons of the adult central nervous system in the turtle. Neurosci. Lett. 32: 510 .

Johnston, R. N., and N. K. Wessells (1980) Regulation of the elongating nerve fibre. Curr. Top. Dev. Biol. 16: 165-206.

Kirschner, M. W. (1980) Implication of treadmilling for the stability and polarity of actin and tubulin polymers in vivo. J. Cell Biol. 86: 330-334.

Landis, S. C. (1983) Neuronal growth cones. Annu. Rev. Physiol. 45: $567-580$

Lasek, R. J., and P. N. Hoffman (1976) The neuronal cytoskeleton, axonal transport and axonal growth. Cold Spring Harbor Conf. Cell Proliferation 3: 10211049

Lasek, R. J., and M. L. Shelanski (1981) Cytoskeleton and the architecture of nervous systems. Neurosci. Res. Program Bull. 19: 1-154.

Letourneau P. C. (1982) Analysis of microtubule number and length in cytoskeletons of cultured sensory neurons. J. Neurosci. 2: 806-814.
Letourneau, P. C. (1983) Differences in the organization of actin in the growth cones compared with the neurites of cultured neurons from chick embryos. J. Cell Biol. 97: 963-973.

Letourneau, P. C., and A. H. Ressler (1984) Inhibition of neurite initiation and growth by taxol. J. Cell Biol. 98: 1355-1362.

Nakai, J. (1956) Dissociated dorsal root ganglia in tissue culture. Am. J. Anat. 99: 81-130.

Nemhauser, I., J. Joseph-Silverstein, and W. D. Cohen (1983) Centrioles as microtubule-organizing centres for marginal bands of molluscan erythrocytes. J Cell. Biol. 96: 979-989.

Ochs, S. (1972) Fast transport of materials in mammalian nerve fibres. Science 176: 252-260.

Osborn, M., R. E. Webster, and K. Weber (1978) Individual microtubules viewed by immunofluorescence and electron microscopy in the same PtK2 cell. J. Cell Biol. 77: R27-R34.

Peters, A., S. L. Palay, and H. F. Webster (1976) The Fine Structure of the Nervous System: The Neurons and Supporting Cells, W. B. Saunders Co., Philadelphia.

Pfenninger, K. H., and M. P. Johnson (1983) Membrane biogenesis in the sprouting neuron. I. Selective transfer of newly synthesized phospholipid into the growing neurite. J. Cell. Biol. 97: 1038-1042.

Pfenninger, K. H., and M. F. Maylie-Pfenninger (1981) Lectin labeling of sprouting neurons. II. Relative movement and appearance of glycoconjugates during plasmalemmal expansion. J. Cell Biol. 89: $547-559$.

Ramón y Cajal, S. (1972) The Structure of the Retina, S. A. Thorpe and M. Glickstein, transl., pp. 140-152, Charles C Thomas, Publisher, Springfield, IL.

Schliwa, M., and J. van Blerkom (1981) Structural interaction of cytoskeletal components. J. Cell Biol. 90: 222-233.

Schwartz, J. H., J. E. Goldman, R. Aubron, and D. J. Goldberg (1976) Axonal transport of vesicles carrying $3-\mathrm{H}$ serotonin in the metacerebral ganglion of Aplysia californica. Cold Spring IIarbor Symp. Quant. Biol. 40: 83-92.

Sharp, G. A., K. Weber, and M. Osborn (1982) Centriole number and process formation in established neuroblastoma cells and primary dorsal root ganglion neurones. Eur. J. Cell. Biol. 29: 97-103.

Shaw, G., M. Osborn, and K. Weber (1981) Arrangement of neurofilaments, microtubules and microfilament-associated proteins in cultured dorsal root ganglia cells. Eur. J. Cell Biol. 24: 20-27.

Spiegelman, B. M., M. A. Lopata, and M. W. Kirschner (1979) Aggregation of microtubule initiation sites preceding neurite outgrowth in mouse neuroblastoma cells. Cell 16: 253-263.

Spooner, B. S., and C. R. Holladay (1981) Distribution of tubulin and actin in neurites and growth cones of differentiating nerve cells. Cell Motil. 1: 167-178.

Summers, K., and M. W. Kirschner (1979) Characteristics of the polar assembly and disassembly of microtubules observed in vitro by darkfield light miscroscopy. J. Cell Biol. 83: 205-217.

Telzer, B. R., and J. L. Rosenbaum (1980) Cell-cycle dependent in vitro assembly of microtubules onto the pericentriolar material of HeLa cells. J. Cell Biol. 81: 484-501.

Thoenen, H. T. and G. W. Kreutzberg (1981) The role of fast transport in the nervous system. Neuroscience Res. Bull. 20:1-138.

Tosney, K. W., and N. K. Wessells (1983) Neuronal motility: The ultrastructure of veils and microspikes correlates with their motile activities. J. Cell Sci. 61: 389-411.

Tsui, H. T., and W. L. Klein (1983) Hair-pin loops of microtubules at neuronal growth cones. Soc. Neurosci. Abstr. 9: 205.

Tsui, H. T., H. Ris, and W. L. Klein (1983) Ultrastructural networks in growth cones and neurites of cultured central nervous system neurons. Proc. Natl. Acad. Sci. U. S. A. 80: 5779-5783.

Westrum, L. E., E. G. Gray, R. D. Burgoyne, and J. Barron (1983) Synaptic development and microtubule organization. Cell Tissue Res. 231: 93-102

Yamada, K. M., B. S. Spooner, and N. K. Wessells (1970) Axon growth: Roles of microfilaments and microtubules. Proc. Natl. Acad. Sci. U. S. A. $66: 1206-1212$.

Yamada, K. M., B. S. Spooner, and N. K. Wessells (1971) Ultrastructure and function of growth cones and axons of cultured nerve cells. J. Cell Biol. 49: 614-635. 